\title{
Fractional order modelling of fractional-order holds
}

\author{
J.A. Tenreiro Machado
}

\begin{abstract}
Discrete time control systems require sample-and-hold circuits to perform the conversion from digital to analog. Fractional-Order Holds (FROHs) are an interpolation between the classical zero and first order holds and can be tuned to produce better system performance. However, the model of the FROH is somewhat hermetic and the design of the system becomes unnecessarily complicated. This paper addresses the modelling of the FROHs using the concepts of Fractional Calculus (FC). For this purpose, two simple fractional-order approximations are proposed whose parameters are estimated by a genetic algorithm. The results are simple to interpret, demonstrating that FC is a useful tool for the analysis of these devices.
\end{abstract}

Keywords Fractional calculus · Fractional-order holds · Genetic algorithms · Control

\section{Introduction}

Fractional calculus (FC) is a generalisation of the classical theory of differential calculus and deals with integrals and derivatives of non-integer, or even complex, order [1-6]. A huge range of potential applications emerges by embedding the FC concepts with physics and engineering, and presently active research is being pursued [7-18]. In spite of this state of affairs, the formalism of FC is not yet widely known, there remain many phenomena whose study can be more deeply accomplished in the light of FC. The area of dynamical systems and control has received considerable attention. Recently several papers addressing evolutionary concepts and fractional algorithms were proposed. Therefore, embedding the concepts of FC, evolutionary optimisation and control design is clearly a fruitful research strategy.

Fractional-Order Holds (FROHs) are a technique for improving the performance of digital control systems [19-35]. Standard discrete time systems adopt Zero-Order Holds (ZOHs) that model the signal reconstruction done by a conventional digital-to-analog converter (DAC). The $\mathrm{ZOH}$ model describes the effect of converting a discrete time control signal into a continuous time version of it, by holding each sample value for one sample interval. Eventually, the FirstOrder Holds (FOHs) can be used that reconstruct the signal as a piecewise linear approximation of the original sampled signal. FROHs are a compromise between the two aforementioned holding circuits. Such circuits are more complicated than the $\mathrm{ZOHs}$ or the $\mathrm{FOHs}$, but we can take advantage of the extra parameter beta that can be tuned to optimise the system performance.

While the name "Fractional" occurs both for FC and $\mathrm{FROH}$, the fact is that the two areas are apparently unrelated. In this paper, we study the FROH in 
the light of FC, demonstrating that we can develop a fractional-order model of the FROH, leading to a simple analytical representation and easing its application in digital control systems.

Bearing these ideas in mind, this paper is organised as follows. Section 2 introduces the fundamental concepts behind FC and the FROH. Section 3 analyses the frequency response of sample-and-hold circuits and develops simple fractional modelling approximations. The implications upon discrete-time control systems are discussed. Finally, Sect. 4 outlines the main conclusions.

\section{Fundamental concepts}

\subsection{Fractional calculus}

The Riemann-Liouville, Grünwald-Letnikov, and Caputo definitions of fractional derivatives or order $\alpha$ are given by:

$$
\begin{aligned}
{ }_{a}^{\mathrm{RL}} D_{t}^{\alpha} f(t) & =\frac{1}{\Gamma(n-\alpha)} \frac{d^{n}}{d t^{n}} \int_{a}^{t} \frac{f(\tau)}{(t-\tau)^{\alpha-n+1}} d \tau, \\
n-1<\alpha & <n, \\
{ }_{a}^{\mathrm{GL}} D_{t}^{\alpha} f(t) & =\lim _{h \rightarrow 0} \frac{1}{h^{\alpha}} \sum_{k=0}^{\left[\frac{t-a}{h}\right]}(-1)^{k}\left(\begin{array}{l}
\alpha \\
k
\end{array}\right) f(t-k h), \\
{ }_{a}^{C} D_{t}^{\alpha} f(t) & =\frac{1}{\Gamma(n-\alpha)} \int_{a}^{t} \frac{f^{(n)}(\tau)}{(t-\tau)^{\alpha-n+1}} d \tau, \\
n-1<\alpha & <n,
\end{aligned}
$$

where $\Gamma(\cdot)$ is Euler's gamma function, $[x]$ means the integer part of $x$, and $h$ is the time increment.

Using the Laplace transform, we have the expression

$$
\begin{aligned}
\mathcal{L}\left\{{ }_{0}^{\mathrm{RL}} D_{t}^{\alpha} f(t)\right\}= & s^{\alpha} \mathcal{L}\{f(t)\} \\
& -\sum_{k=0}^{n-1} s^{k}{ }_{0} D_{t}^{\alpha-k-1} f 0^{+},
\end{aligned}
$$

where $s$ and $\mathcal{L}$ represent the Laplace variable and operator, respectively.

The Mittag-Leffler function $E_{\alpha}(x)$ is defined by the expression

$E_{\alpha}(x)=\sum_{k=0}^{\infty} \frac{x^{k}}{\Gamma(\alpha k+1)}$.

The Mittag-Leffler function constitutes an "interpolation" between a purely exponential law that occurs in phenomena governed by integer order dynamics and a power law behaviour that occurs in phenomena exhibiting fractional dynamics. In particular, when $\alpha=1$ we have $E_{1}(x)=e^{x}$. An important characteristic of the Mittag-Leffler function is its asymptotic behaviour and, for large values of $x$, we can write

$$
E_{\alpha}(-x) \approx \frac{1}{\Gamma(1-\alpha)} \frac{1}{x}, \quad \alpha \neq 1,0<\alpha<2 .
$$

The Laplace transform results in

$$
\left.\mathcal{L}\left\{E_{\alpha} \pm a t^{\alpha}\right)\right\}=\frac{s^{\alpha-1}}{s^{\alpha} \mp a} .
$$

We verify a natural extension of the Laplace transform pairs from the exponential function, in terms of integer powers of $s$, towards the Mittag-Leffler function, with fractional powers of $s$.

\subsection{Sample and hold circuits}

In a digital system, the $\mathrm{ZOH}$ is a circuit that inverts the sampling process: the value of the impulse signal $u\left(k T_{S}\right)$ is held on the output during the sampling period. Therefore, the output of a $\mathrm{ZOH}$ is a staircase approximation to the original waveform:

$$
\begin{aligned}
u_{\mathrm{ZOH}}(t) & =u\left(k T_{s}\right), \\
k T_{s} \leq t & <(k+1) T_{s}, k=0,1, \ldots
\end{aligned}
$$

In the Laplace domain, the transfer function for a $\mathrm{ZOH}$ yields

$$
G_{\mathrm{ZOH}}(s)=\frac{1-e^{-T_{s}}}{s} .
$$

The $\mathrm{ZOH}$ is the simplest reconstruction circuit and (like the other circuits to be addressed) assumes zero processing delay in converting between digital to ana$\log$.

The FOH takes the slope of the input signal from $t=(k-1) T_{s}$ up to $t=T_{s}$ and uses it to make a guess as to where the output waveform is going to be at instant $t=(k+1) T_{s}$. Therefore, the FOH draws a straight line from the current position to the expected future position, as the output of the waveform:

$$
\begin{gathered}
\left.u_{\mathrm{FOH}}(t)=u\left(k T_{s}\right)+\left(t-k T_{s}\right)\left\{u\left(k T_{s}\right)-u(k-1) T_{s}\right]\right\}, \\
k T_{s} \leq t<(k+1) T_{s} .
\end{gathered}
$$

The Laplace transfer function of the FOH is

$$
G_{\mathrm{FOH}}(s)=\frac{1+s T_{s}}{T_{s}}\left(\frac{1-e^{-T_{s}}}{s}\right)^{2} .
$$


Nevertheless, the next value of the output signal will probably not be the same as the expected value and, therefore, the FOH may also produce some discontinuities.

The FROH establishes a compromise between the $\mathrm{ZOH}$ and the FOH. The FROH acts like an interpolation between the other two holding circuits and takes an adjustable parameter $\beta$ whose value is to be determined by some tuning method

$$
\begin{aligned}
u_{\mathrm{FROH}}(t)= & u\left(k T_{s}\right)+\beta \frac{t-k T_{s}}{T_{s}}\left\{u\left(k T_{s}\right)\right. \\
& \left.\left.-u(k-1) T_{s}\right]\right\}, \\
k T_{s} \leq t< & (k+1) T_{s} .
\end{aligned}
$$

The Laplace transfer function of the FROH then results in

$G_{\mathrm{FROH}}(s)=\frac{1-e^{-T_{s}}}{s} 1+\beta \frac{1-e^{-T_{s}}\left(1+s T_{s}\right)}{s T_{s}}$.
The FROH is more complicated than the other hold circuits, but may lead to a better system performance after tuning parameter $\beta$.

\section{A fractional model of the FROH}

We start by analysing the performance of a simple digital control system under the action of the FROH. The results motivate the development of a model having in mind the concepts of FC.

\subsection{Closed loop time response}

Let us consider the closed loop system represented in Fig. 1 where $G_{c}(s)$ represents the controller and $G(s)$ the plant. We adopt a unit step reference input $R(s)=\frac{1}{s}$ and a plant with transfer function $G(s)=$ $\frac{1}{(s+1)^{3}}$. The controller is considered to be a simple PID, $u(t)=k_{p}\left[e(t)+\frac{1}{T_{i}} \int e(t) d t+T_{d} \frac{d e(t)}{d t}\right]$, tuned
Fig. 1 Simple closed loop control system

Fig. 2 Closed loop time response for $\beta=\{-1,-0.5,0,0.5,1\}$ and $T_{s}=0.01$
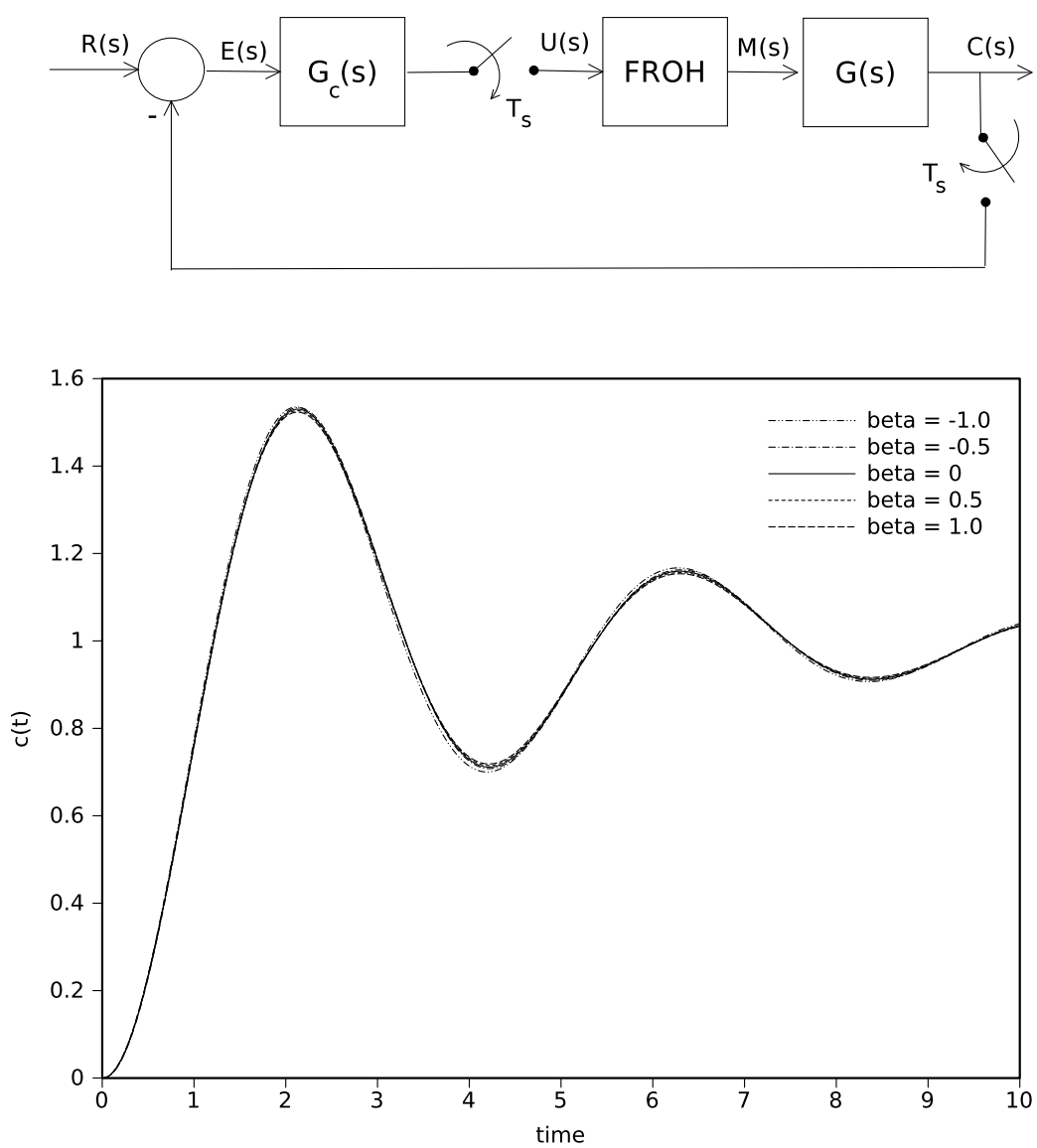
Fig. 3 Closed loop time response for $\beta=\{-1,-0.5,0,0.5,1\}$ and $T_{s}=0.1$
Fig. 4 Polar plots of the FROH for

$\beta=\{-1,-0.5,0,0.5,1\}$, $0 \leq \omega<\pi$
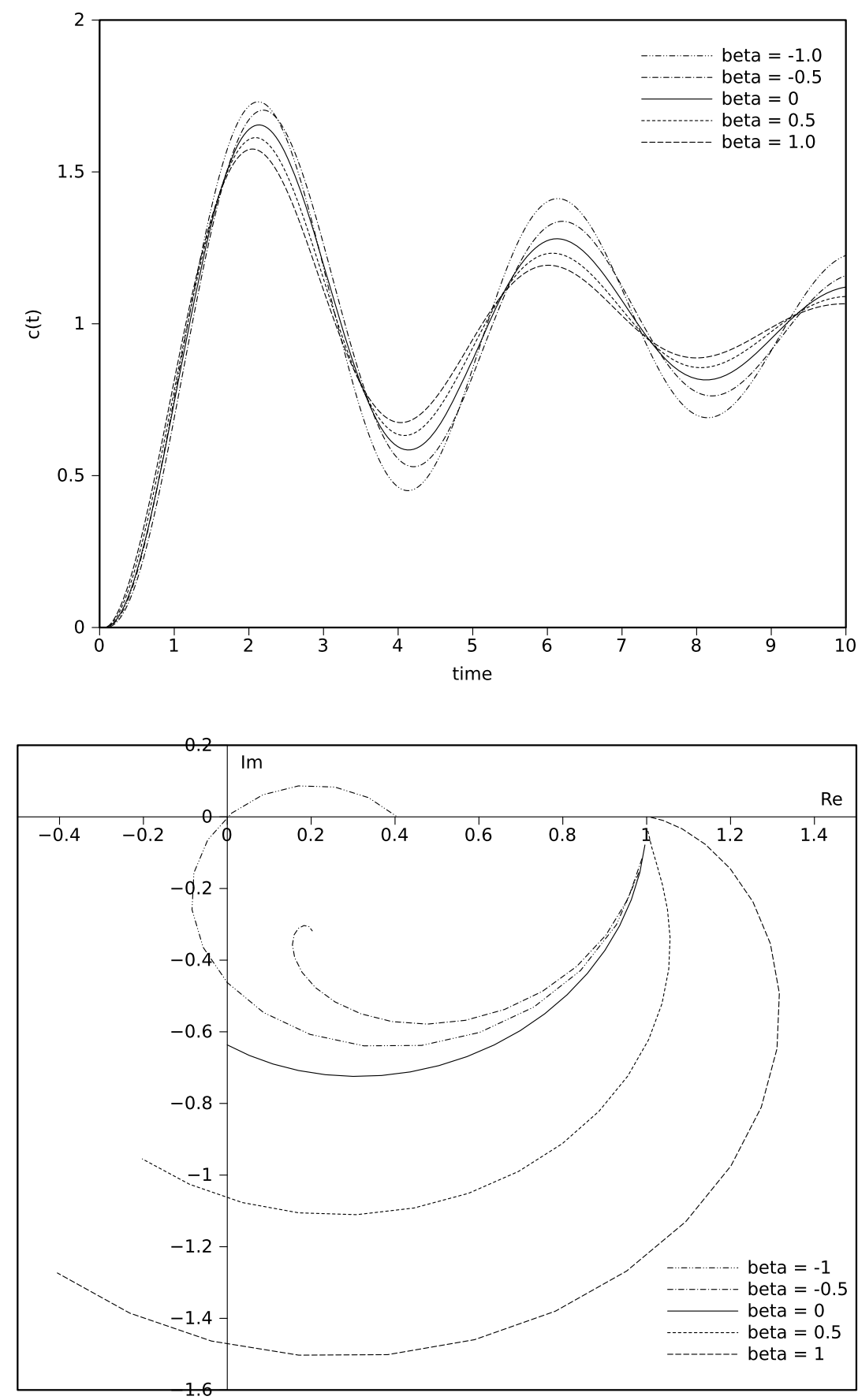

according to the Ziegler-Nichols open-loop heuristics that leads to $k_{p}=5.494, T_{i}=1.612, T_{d}=0.403$ and to $G_{c}(s)=\frac{2.214 s^{2}+5.494 s+3.408}{s}$. For the experiments with the FROH, the cases $\beta=\{-1,-0.5,0,0.5,1\}$ are tested, and the sampling periods $T_{s}=\{0.01,0.1\}$ are depicted in Figs. 2 and 3.
The $\mathrm{ZOH}$ and the $\mathrm{FOH}$ correspond to the cases of $\beta=0$ and $\beta=1$, respectively. Furthermore, we verify that the dynamic compensation promoted by the FROH when tuning $\beta$ is mainly visible for high sampling periods since responses become close to each other for fast sampling rates. 
Fig. 5 Polar plots of the FROH frequency response and the parameters of the two approximate

FC-models for $\beta=0.5$, $0 \leq \omega<\pi$
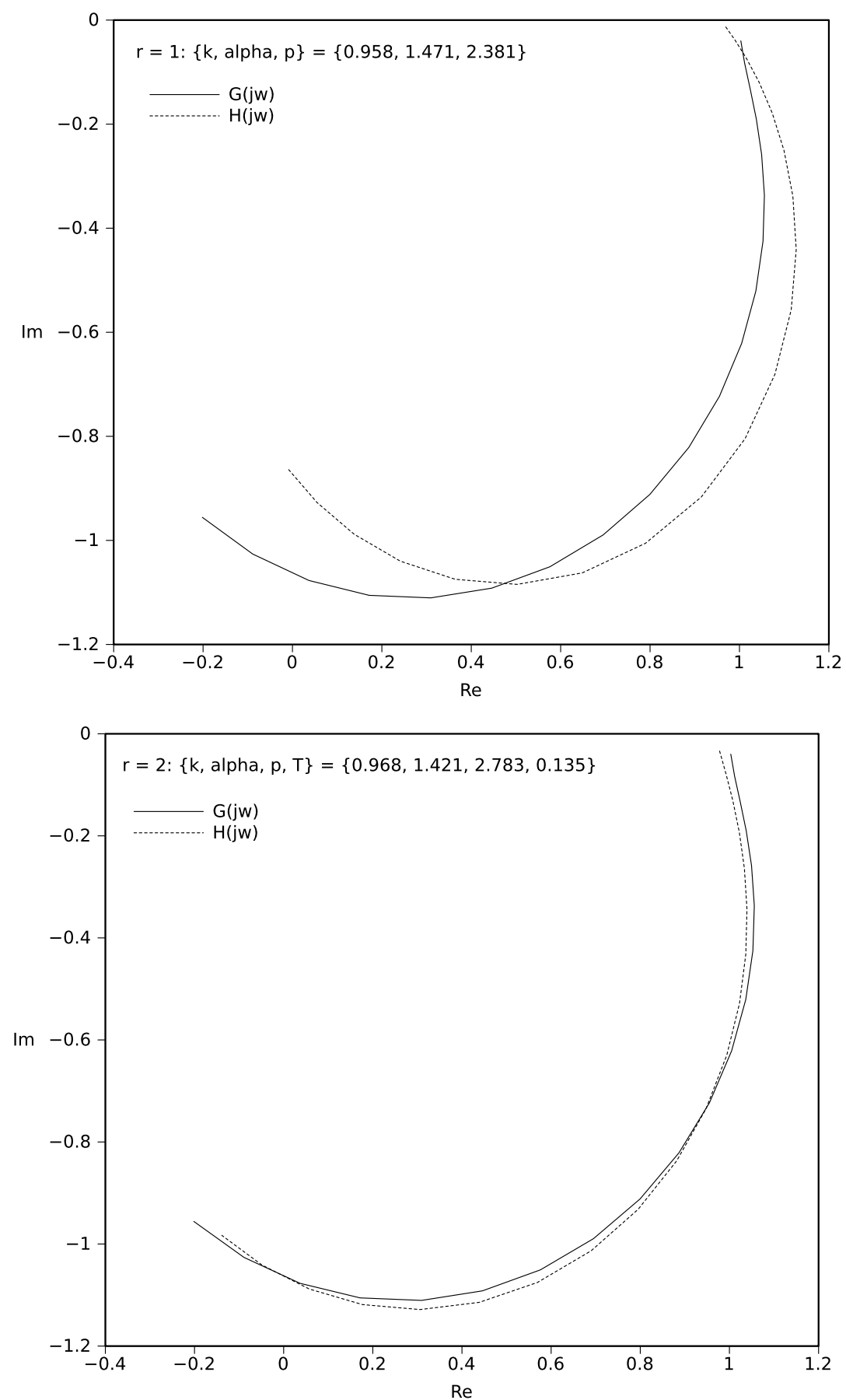

\subsection{Fractional order model}

In this sub-section, we develop an alternative model of the FROH based on FC concepts. We start by considering the FROH Laplace model (13) and $s=j \omega$, $j=\sqrt{-1}$, that is, the frequency response. For exam- ple, when $T_{s}=1$, we get the polar plot represented in Fig. 4 for $0 \leq \omega<\pi$.

The plot shows strong resemblance with those of fractional low pass filters. This observation motivates the quest for an approximate model that, by incorporating the FC concepts, can lead to a simpler, but 
Fig. 6 Variation of the two FROH FC-model parameters versus $\beta=\{-1,-0.5,0,0.5,1\}$ for $0 \leq \omega<\pi$
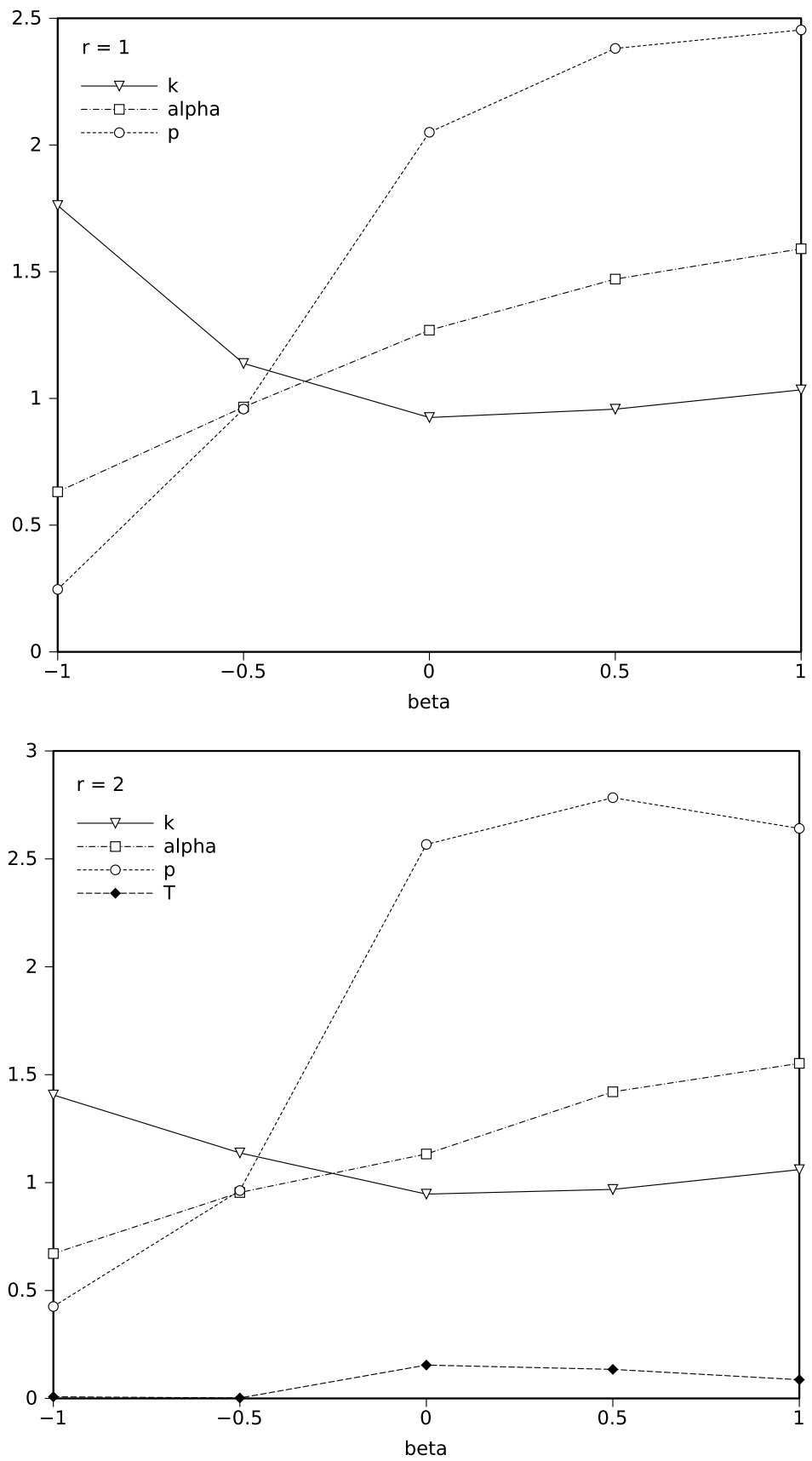

more insightful, model than expression (13). Several FC-models were tested having in mind a compromise between simplicity and accuracy. The choice for the FC-models fell upon the two cases:

$H_{\mathrm{FROH}}^{1}(s)=\frac{k}{1+\left(\frac{s}{p}\right)^{\alpha}}$,

$$
H_{\mathrm{FROH}}^{2}(s)=\frac{k e^{-s T}}{1+\left(\frac{s}{p}\right)^{\alpha}},
$$

where $k, p, \alpha$ and $T$ denote the gain, pole, fractional order and time delay, respectively. These models are very simple, but, on the other hand, lead to reasonable accuracy. 
For adjusting $H_{\mathrm{FROH}}^{r}(j \omega), r=1,2$, to the numerical data, a standard genetic algorithm is adopted with elitism, crossover within all population and $5 \% \mathrm{mu}-$ tation probability. Several experiments demonstrated that the best fitness function $J$ is given by

$J=\frac{1}{N} \sum_{i=1}^{N} \frac{\left|G_{\mathrm{FROH}}\left(j \omega_{i}\right)\right|-\left|H_{\mathrm{FROH}}^{r}\left(j \omega_{i}\right)\right|}{\left|G_{\mathrm{FROH}}\left(j \omega_{i}\right)\right|+\left|H_{\mathrm{FROH}}^{r}\left(j \omega_{i}\right)\right|}$,

where $N$ represents the total number of frequency points. This fitness function leads to good results since, by calculating the ratio between the difference and the sum of the two values, one captures the relative error of the adjustment, avoiding "saturation-like" effects that occur when using the standard Euclidean norm due to the simultaneous presence of large and small values. In the calculations, we used $N=20$, a population of 5000 individuals and 5000 iterations of the genetic algorithm.

Figure 5 shows the polar plots for $r=1,2$ with superposition of the original frequency response (13) and the resulting from (14)-(15) when $\beta=0.5$. We verify a good match between the two traces, namely for the case of the second model that includes the time delay.

Figure 6 reveals the smooth variation of the parameters $\{k, p, \alpha\}, r=1$, and $\{k, p, \alpha, T\}, r=2$, versus $\beta=\{-1,-0.5,0,0.5,1\}$, when $0 \leq \omega<\pi$. As expected, the gain is always very close to one and the output smoothness accomplished when passing from the $\mathrm{ZOH}$ to the $\mathrm{FOH}$ is reflected upon the value of $\beta$ that increases proportionally, but the bandwidth is preserved because the pole is shifted to higher frequencies. In the case of the second model, the delay $T$ has always very small values.

\section{Conclusions}

FROHs constitute a simple technique for improving the performance of digital control systems particularly for the case of low sampling frequencies. Nevertheless, the expressions usually adopted for modelling this digital-to-analog conversion are complicated and lead to a limited insight into the sample-and-hold dynamics. This paper analysed the FROHs in the light of FC. For this purpose, the feasibility of two simple models including an explicit fractional order pole was evaluated. To adjust the values numerically, a genetic algorithm and a fitness function that measures the relative error of the approximation were adopted. The approximation scheme leads to simple and easily interpretable results that simplify the design of closed loop digital control including the FROH.

\section{References}

1. Oldham, K., Spanier, J.: The Fractional Calculus: Theory and Application of Differentiation and Integration to Arbitrary Order. Academic Press, New York (1974)

2. Miller, K., Ross, B.: An Introduction to the Fractional Calculus and Fractional Differential Equations. Wiley, New York (1993)

3. Samko, S., Kilbas, A., Marichev, O.: Fractional Integrals and Derivatives: Theory and Applications. Gordon and Breach Science Publishers, London (1993)

4. Podlubny, I.: Fractional Differential Equations. Academic Press, San Diego (1999)

5. Kilbas, A., Srivastava, H.M., Trujillo, J.: Theory and Applications of Fractional Differential Equations. Elsevier, Amsterdam (2006)

6. Machado, J.T., Kiryakova, V., Mainardi, F.: Recent history of fractional calculus. Commun. Nonlinear Sci. Numer. Simul. 16(3), 1140-1153 (2011)

7. Oustaloup, A.: La Commande CRONE: Commande Robuste D'Ordre Non Entier. Hermès, Paris (1991)

8. Zaslavsky, G.: Hamiltonian Chaos and Fractional Dynamics. Oxford University Press, New York (2005)

9. Magin, R.: Fractional Calculus in Bioengineering. Begell House Publishers, Redding (2006)

10. Diethelm, K.: The Analysis of Fractional Differential Equations. Springer, Berlin (2010)

11. Mainardi, F.: Fractional Calculus and Waves in Linear Viscoelasticity: An Introduction to Mathematical Models. Imperial College Press, London (2010)

12. Povstenko, Y.: Signaling problem for time-fractional diffusion-wave equation in a half-space in the case of angular symmetry. Nonlinear Dyn. 59(4), 593-605 (2010)

13. Zeng, C., Yang, Q., Wang, J.: Chaos and mixed synchronization of a new fractional-order system with one saddle and two stable node-foci. Nonlinear Dyn. 65(4), 457-466 (2011)

14. Machado, J.T.: And I say to myself: "What a fractional world!” J. Fract. Calc. Appl. Anal. 14(4), 635-654 (2011)

15. Balochian, S., Sedigh, A.K., Haeri, M.: Stabilization of fractional order systems using a finite number of state feedback laws. Nonlinear Dyn. 66(1-2), 141-152 (2011)

16. Herzallah, M.A., Muslih, S.I., Baleanu, D., Rabei, E.M.: Hamilton-Jacobi and fractional like action with time scaling. Nonlinear Dyn. 66(4), 549-555 (2011)

17. Gao, Z., Liao, X.: Rational approximation for fractionalorder system by particle swarm optimization. Nonlinear Dyn. 67(2), 1387-1395 (2012)

18. Cafagna, D., Grassi, G.: Observer-based projective synchronization of fractional systems via a scalar signal: application to hyperchaotic Rössler systems. Nonlinear Dyn. 68(1-2), 117-128 (2012) 
19. Passino, K., Antsaklis, P.J.: Reconstruction methods to obtain inverse stable sampled systems. In: Proceedings of the 1985 American Control Conference, Boston, MA, pp. 964969 (1985)

20. Passino, K., Antsaklis, P.J.: Inverse stable sampled low-pass systems. Int. J. Control 47(6), 1905-1913 (1988)

21. Ishitobi, M.: Stability of zeros of sampled system with fractional order hold. IEE Proc., Control Theory Appl. 143(3), 296-300 (1996)

22. Sheen, I.E., Tsai, J.S., Shieh, L.S.: Optimal digital redesign of continuous-time systems using fractional-order hold. Optim. Control Appl. Methods 18(6), 399-422 (1997)

23. Bárcena, R., De la Sen, M.: On the sufficiently small sampling period for the convenient tuning of fractional-order hold circuits. IEE Proc., Control Theory Appl. 150(2), 183188 (2003)

24. Yuz, J.I., Goodwin, G.C., Garnier, H.: Generalised hold functions for fast sampling rates. In: 43rd IEEE Conference on Decision and Control, Atlantis, Bahamas, vol. 2, pp. 1908-1913 (2004)

25. De la Sen, M., Bilbao, M.: Optimization of the correcting gains of fractional order hold devices for inter-sample output reconstruction. Am. J. Appl. Sci. 2(4), 771-773 (2005)

26. De la Sen, M., Bilbao-Guillerna, A.: Fractional hold circuits versus positive realness of discrete transfer functions. Discrete Dyn. Nat. Soc. 2005(3), 373-378 (2005)

27. De la Sen, M.: About optimal fractional hold circuits for inter-sample output reconstruction in sampled-data systems. Sensors 7(12), 3146-3155 (2007)
28. B'arcena, R., Etxebarria, A.: Adaptive control of printing devices using fractional-order hold circuits adjusted through neural networks. J. Franklin Inst. 344(6), 801-812 (2007)

29. Królikowski, A., Horla, D.: Application of LMI for design of digital control systems. Bull. Pol. Acad. Sci., Tech. Sci. 56(4), 361-365 (2008)

30. Ugalde, U., B'arcena, R., Basterretxea, K.: Ripple-free control response by using a fractional order hold device. WSEAS Trans. Syst. 7(7), 679-688 (2008)

31. Basterretxea, K., Bárcena, R., Ugalde, U.: Design and synthesis of a configurable fractional order hold device for sampled-data control systems. WSEAS Trans. Circ. Syst. 7(8), 869-878 (2008)

32. Bárcena, R., Etxebarria, A.: Mitigating the effect of fractional time delays in hybrid control systems. IET Control Theory Appl. 2(1), 24-31 (2008)

33. Zhang, G., Chen, X., Chen, T.: $l_{p}$-equivalence of discretizations of analog controllers. In: Proceedings of the 17th IFAC World Congress 15232-15237, Seoul, Korea (2008)

34. Alonso-Quesada, S., De la Sen, M.: Robust adaptive stabilization of linear time-invariant dynamic systems by using fractional-order holds and multirate sampling controls. Discrete Dyn. Nature Soc. (2010). doi:10.1155/2010/620546

35. Nishi, M., Ishitobi, M.: Sampled-data models for affine nonlinear systems using a fractional-order hold and their zero dynamics. Artif. Life Robot. 15(4), 500-503 (2010) 